\title{
New Constructions in Local Quantum Physics*
}

\author{
Bert Schroer \\ CBPF, Rua Dr. Xavier Sigaud 150 \\ 222-180 Rio de Janeiro, Brazil \\ and Institut fuer Theoretische Physik der FU-Berlin
}

January 4, 2005

\begin{abstract}
Among several ideas which arose as consequences of modular localization there are two proposals which promise to be important for the classification and construction of QFTs. One is based on the observation that wedge-localized algebras may have particle-like generators with simple properties and the second one uses the structural simplification of wedge-localized algebras in the holographic lightfront projection. Factorizable $\mathrm{d}=1+1$ models permit to analyse the interplay between particle-like aspects and chiral field properties of lightfront holography.

Pacs 11.10.-z, 11.55.-m
\end{abstract}

\section{How modular theory entered particle physics}

The following introductory remarks about the history and the physical content of modular theory are intended to be helpful to understand the recent role of modular localization in the classification and construction of models of QFT without the use of Lagrangian quantization.

\subsection{Remarks about history of modular localization}

The beginnings of modular theory date back to the second half of the 1960s when two independent ideas, one from mathematics and one from particle physics, merged together 11. On the mathematical side the Japanese mathematician Tomita generalized a concept, which before was only studied in the special context of the Haar measure ("unimodular") in group algebra theory, to the general setting of von Neumann algebras. At the same time three physicists, Haag, Hugenholtz and Winnink 2, found a conceptual framework for the direct field theoretic description of the thermodynamic limit ("open systems") in terms

*Talk presented at "Rigorous quantum field theory", symposium in honor of J. Bros, Paris, July 2004 
of operator algebras and their commutants [3]. Their important contribution, which became immediately incorporated by Takesaki into Tomita's modular theory, was the realization that the KMS condition (introduced by Kubo, Martin and Schwinger as a computational tool) acquired a fundamental conceptual significance in their new thermal setting.

It took another decade in order to appreciate the geometric significance of this modular formalism for the problem of localization of algebras and states in QFT [5]. This was preceded by an important mathematical application in the classification of type III von Neumann algebras by A. Connes [] and followed up by a theorem of Driessler [7] stating that wedge-localized algebras are factors of type $\mathrm{III}_{1}$. As a consequence double cone algebras in conformal invariant theories inherit this property ${ }^{1}$. Later refinements supported the idea that compactly localized subalgebras in QFT are isomorphic to the unique hyperfinite type $\mathrm{III}_{1}$ factor. For more detailed reviews of modular theory from the mathematical physics viewpoint we refer to [1] 8, 9]

Although hyperfinite type III $_{1}$ algebras appear at first sight (as a result of this uniqueness) in a certain sense as void of structure as points in geometry, they are in other aspects much richer since they contain subalgebras of all types and one can form nontrivial intersections from copies placed into different positions within a common Hilbert space $H$. In fact we know from later developments of algebraic QFT that the full richness of a model of QFT is encoded in the notion of a net of spacetime-indexed von Neumann algebras as subalgebras of $B(H)$ 3.

The net result of this thread of ideas, which culminated in the mathematical identification of simple building blocks of QFT, is interesting from many viewpoints. From a philosophical standpoint it tells us that the algebraic aspects of QFT comply perfectly with Leibniz's dictum that reality emerges from the relation between indecomposable entities ("monades") and not from their individual position with respect to an absolute outside reference.

This is not the first time that philosophical ideas of Leibniz became relevant in physics. In Einstein's "hole argument" [4] it played a significant role in the birth of general relativity; in particular it helped Einstein (and independently Hilbert) to overcome a misconception about how the local covariance of the Einstein-Hilbert field equations and the Newtonian limit fit together. By upholding the local covariance principle, i.e. the idea that local isomorphism classes of isometric diffeomorphisms replace the global notion of an absolute Minkowski spacetime inertial frame of special relativity, Einstein realized that his difficulties to obtain agreement with the Newtonian limit came from a computational misconception.

In fact it was shown recently that the Leibniz viewpoint of physical reality emerging from relations between entities rather than from positions in a preassigned absolute "inertia ether" can actually be extended in order to combine the quantum algebraic modular aspects with the classical covariance principle

\footnotetext{
${ }^{1}$ In conformal theories double cone algebras are conformally equivalent to wedge algebras and therefore inherit the hyperfinite typ $\mathrm{III}_{1}$ property.
} 
into a "local (quantum) covariance principle" [10. This places QFT in CST much closer to a still elusive background-independent quantum gravity than ever before.

In the following we will argue that the "monades" of QFT are the wedge localized algebras which (thanks to Driessler's work [7]) are known to be isomorphic copies of hyperfinite type $\mathrm{III}_{1}$ factor. In order to avoid lengthy terminology we will refer to the basic hyperfinite type $\mathrm{III}_{1}$ von Neumann algebra as the monade algebra (MA). The most convincing affirmation of this way of viewing QFT as arising from wedge localized MA is the fact that models of quantum field theory can be completely specified by positioning a finite number of operator algebra copies of the MA into suitably chosen relative positions within a common Hilbert space $^{2}$ [1].

This way of looking at QFT permits a particularly natural intrinsic formulation in low dimensional QFT. In the case of $d=1+1$ the "modular inclusion" of two MA specifies all data needed to characterize a specific QFT in terms of the structure of its Poincaré covariant nets and for $\mathrm{d}=1+2$ one achieves a complete characterization in terms of "modular intersection" of three MA. Modular positions which are associated with a characterization of higher dimensional QFT models are also known [1], but in their present formulation they appear less natural i.e. more concocted in order to generate the desired Poincaré symmetry structure of Minkowski spacetime.

Accepting the philosophical, conceptual and mathematical implications, one may ask the question whether this approach guided by Leibniz's philosophy is just an esoteric new way of looking at particle physics or if it also has constructive clout, i.e. whether one can actually classify models of QFT and elaborate a realistic scenario of their construction along those lines. Admittedly the apparent simplicity of generating QFT from the positioning of a finite number of MAs is somewhat deceiving; the problem of an intrinsically formulated positioning of MAs is actually quite hard since the appropriate concepts and mathematical tools are to a large extend still missing. Already the characterization of one $\mathrm{MA}$ in Hilbert space i.e. in the setting of local quantum physics of massive particles the description $A(W) \subset_{P} B(H)$ is a difficult problem; here $A(W)$ denotes a wedge localized MA, $B(H)$ is the algebra of bounded operators on Fock space of massive particles obtained by scattering theory (assuming asymptotic completeness) and the subscript $P$ indicates that the inclusion is meant in the extended sense that also the action of the Poincaré group on it (which creates a family of wedge localized MAs) is known.

There are two situations in which this positioning of the MA is reasonably simple and the construction of the net (and its generating pointlike field coordinatizations) can actually be carried out. These are the interactionless theories whose one particle components are described in terms of Wigner representations of elementary systems and $d=1+1$ factorizing models. For general interacting theories the idea of lightfront holography is very helpful because it suggests to

${ }^{2}$ We will use the terminology MA also for the positioned operator algebra copies of the basic MA. 
classify and construct wedge algebras in terms of their lightfront holographies. These problems will be addressed in this paper.

In the remainder of this introduction the modular approach to the interactionfree QFTs will be given; as a result of its simplicity it also serves well as a pedagogical introduction into the setting of modular localization.

\subsection{Modular construction of interaction-free QFT}

This construction via modular localization proceeds in three steps as follows 12] 13$] 14]$

1. Fix a reference wedge region, e.g. $W_{R}=\left\{x \in \mathbb{R}^{4} ; x^{1}>\left|x^{0}\right|\right\}$ and use the Wigner positive energy representation of the $W_{R}$-affiliated boost group $\Lambda_{W_{R}}(\chi)$ and the $x^{0}-x^{1}$-reflection $j_{W}{ }^{3}$ along the edge of the wedge $j_{W_{R}}$ in order to define the following antilinear unbounded closable operator (with $\left.\operatorname{clos} S=\operatorname{clos} \Delta^{\frac{1}{2}}\right)$. Retaining the same notation for the closed operators, one defines

$$
\begin{aligned}
& S_{W_{R}}:=J_{W_{R}} \Delta^{\frac{1}{2}} \\
& J_{W_{R}}:=U\left(j_{W_{R}}\right), \Delta^{i t}:=U\left(\Lambda_{W_{R}}(2 \pi t)\right)
\end{aligned}
$$

The commutativity of $J_{W_{R}}$ with $\Delta^{i t}$ together with the antiunitarity of $J_{W_{R}}$ yield the property which characterize a Tomita operator ${ }^{4} S_{W_{R}}^{2} \subset 1$ whose domain is identical to its range. Such operators are completely characterized in terms of their +1 real eigenspaces which in the present context amounts to real standard subspace $K\left(W_{R}\right)$ of the Wigner representation space $H$

$$
\begin{aligned}
K\left(W_{R}\right) & :=\left\{\psi \in H, S_{W_{R}} \psi=\psi\right\} \\
\frac{K\left(W_{R}\right)+i K\left(W_{R}\right)}{} & =H, K\left(W_{R}\right) \cap i K\left(W_{R}\right)=0 \\
J_{R} K\left(W_{R}\right) & =K\left(W_{R}\right)^{\perp}=: K\left(W_{R}\right)^{\prime}
\end{aligned}
$$

$K\left(W_{R}\right)$ is closed in $H$ whereas the complex subspace spanned together with the -1 eigenspace $i K\left(W_{R}\right)$ is the dense domain of the Tomita operator $S_{W_{R}}$ and forms a Hilbert space in the graph norm of $S_{W_{R}}$. The denseness in $H$ of this span $K\left(W_{R}\right)+i K\left(W_{R}\right)$ and the absence of nontrivial vectors in the intersection $K\left(W_{R}\right) \cap i K\left(W_{R}\right)$ is called "standardness". The right hand side in the third line refers to the symplectic complement i.e. a kind of "orthogonality" in the sense of the symplectic form $\operatorname{Im}(\cdot, \cdot)$.

\footnotetext{
${ }^{3}$ The reflection on the edge of the wedge is related to the total TCP reflection by a $\pi$-rotation around the $x_{1}$-axis. Hence in certain cases the irreducible representation has to be doubled. This is always the case for zero mass finite helicity representations and more generally if particles are not selfconjugate.

${ }^{4}$ Operators with this property are the corner stones of the Tomita-Takesaki modular theory 19 of operator algebras. Here they arise in the spatial Rieffel-van Daele spatial setting [16] of modular theory from a realization of the geometric Bisognano-Wichmann situation within the Wigner representation theory.
} 
The application of Poincaré transformations to the reference situation generates a consistent family of wedge spaces $K(W)=U(\Lambda, a) K\left(W_{R}\right)$ if $W=(\Lambda, a) W_{R}$. These subspaces carry a surprising amount of informations about local quantum physics; their structure even preempts the spin-statistics connection by producing a mismatch between the symplectic and the geometric complement ( $W^{\prime}$ denotes the causal complement in terms of Minkowski space geometry) which is related to the spin-statistics factor 13$] 14$

$$
\begin{aligned}
K(W)^{\prime} & =Z K\left(W^{\prime}\right) \\
Z^{2} & =e^{2 \pi i s}
\end{aligned}
$$

Another surprising fact is that the modular setting prepares the ground for the field theoretic on-shell crossing property, since the equation characterizing the real modular localization subspaces in more details reads

$$
\left(J \Delta^{\frac{1}{2}} \psi\right)(p)=V \overline{\psi_{c}(-p)}=\psi(p)
$$

i.e. the complex conjugate of the analytically continued wave function (but now referring to the charge-conjugate situation) is up to a constant matrix $V$ which acts on the spin indices equal to the original wave function.

2. The sharpening of localization is obtained by intersecting wedges in order to obtain real subspaces as causally closed subwedge regions:

$$
K(\mathcal{O}):=\cap_{W \supset \mathcal{O}} K(W)
$$

The crucial question is whether they are "standard". According to an important theorem of Brunetti, Guido and Longo [12] standardness universally holds for spacelike cones $\mathcal{O}=\mathcal{C}$ in all positive energy representations. In case of finite spin/helicity representations the standardness also holds for intersections leading to (arbitrary small) double cones $\mathcal{D}$. In those cases where the double cone localized spaces with pointlike "cores" are trivial (massless infinite spin [17], massive $d=1+2$ anyons [18]), the smallest localization regions are spacelike cones with semiinfinite strings as cores. Without loss of generality one may restrict localization regions to causally complete regions.

3. In the absence of interactions the transition from free particles to localized operator algebras is most appropriately done in a functorial way by applying the Weyl (CCR) (or in case of halfinteger spin the CAR functor) to the localization $\mathrm{K}$-spaces ${ }^{5}$ :

$$
\begin{aligned}
\mathcal{A}(\mathcal{O}) & :=\operatorname{alg}\{W \operatorname{eyl}(\psi) \mid \psi \in K(\mathcal{O})\} \\
W \operatorname{eyl}(f) & :=\operatorname{expi}\left\{a^{*}(\psi)+\text { h.a. }\right\}
\end{aligned}
$$

\footnotetext{
${ }^{5}$ To maintain simplicity we limit our presentation to the bosonic situation and refer to $13 \sqrt{14}$ for the general treatment.
} 
where $a^{\#}(\psi)$ are the creation/annihilation operators of particles in the Wigner wave function $\psi$. The functorial relation between real subspaces and von Neumann algebras preserves the causal localization structure [20] and commutes with the process of improvement of localization through the formation of intersections.

For later purposes we introduce the following definition [21].

Definition 1 A vacuum-polarization-free generator (PFG) for a region $O$ is an operator affiliated with the algebra $\mathcal{A}(\mathcal{O})$ which created a vacuum-polarizationfree one-particle vector

$$
\begin{gathered}
G \eta \mathcal{A}(\mathcal{O}) \\
G \Omega=1-\text { particle }
\end{gathered}
$$

Since these wedge algebra-affiliated operators $G$ are generally unbounded, one has to comment on their domain properties. We will adopt the natural assumptions that they admit dense domains which, similar to smeared Wightman fields, are stable under translations. This definition permits to characterize the presence of interactions by the interaction induced vacuum polarization as a result of the following statement

Proposition 2 The existence of subwedge-localized PFGs characterizes interactionfree theories.

The proof uses the fact that PFGs are on-shell (weak solutions of the KleinGordon equation [33) and subwedge-localized; the analytic argument is completely analogous to that of the theorem about the equality of a two-point function with that of a free field implying the equality of the associated covariant field with a free field [22] 23] (the restriction to covariance and pointlike localization is easily seen to be not necessary). The existence of wedge-localized PFGs $G \eta \mathcal{A}(W)$ is a consequence of modular theory, but their domain $\operatorname{dom}(G)$ is generally not stable under all translations (but only under those translations which transform the wedge into itself). Such PFGs do not admit a Fourier transform i.e. they are not tempered [33. Hence in the presence of interactions the particle localization through the application of localized operators to the vacuum is weakened; according to the previous proposition the QFT cannot localize particles in subwedge regions. Accordingly the functorial relation between particle and field localization breaks down and one has to look for a substitute.

In the next section we will show that the requirement that wedge-localized $G$ fulfill the domain properties of the definition (i.e. are "tempered") leads to an explicit characterization of the associated wedge-localized algebras in terms of a simple algebraic structure of their generators. This amounts to the complete knowledge of the QFT in the sense of its algebraic net. Namely it can be shown that the knowledge of generators of wedge algebras together with the knowledge how Poincaré transformations acts on this reference wedge algebra and generate the family of all wedge algebras in different spacetime position is 
sufficient to build up the complete net of algebras through the formation of intersections of wedge algebras (in analogy to (5)). Examples of tempered wedge localized PFGs are obtained by Fourier transforming generating operators of Zamolodchikov-Faddeev algebras (8) and there are reasons to believe that the $\mathrm{d}+1+1$ factorizing models exhaust the possibilities for tempered PFGs. Knowing the PFG generators explicitly as one does in these models, one can construct the net and its local field generators which are of course much more involved than the non-local wedge generators.

In the third section the idea of lightfront holographic projection will be used in order to classify wedge algebras in terms of extended chiral algebras. The unsolved problems of inverse lightfront holography i.e. the problem of reconstructing ambient algebras from their holographic projections is the main obstacle in the general classification\&construction. Here again the restriction to $\mathrm{d}=1+1$ factorizing models is very helpful.

\section{Modular localization and the bootstrap-formfactor program}

The various past attempts at S-matrix theories which aimed at direct constructions of scattering data without the intermediate use of local fields and local observables provide illustrations of what is meant by an "on-shell" approach to particle physics. The motivation behind such proposals was first spelled out by Heisenberg [25]. It consisted in the hope that by limiting oneself to particles and their mass-shells, one avoids (integration over) fluctuations on a scale of arbitrarily small spacelike distances causing ultraviolet divergencies which at the pre-renormalization days of Heisenberg's S-matrix proposal appeared to be an incurable disease of QFT. The main purpose of staying close to particles and using scattering concepts ("on-shell") is the avoidance of inherently singular objects as pointlike fields in calculational steps This is certainly a reasonable aim independent of whether one believes or not that a formulation of interactions in terms of singular pointlike fields exists for $\mathrm{d}=1+3$ QFT in the mathematical physics sense.

Since the early 1950s, in the aftermath of renormalization theory, the relation between particles and fields received significant elucidation through the derivation of time-dependent scattering theory. In the course of this it also became clear that Heisenberg's S-matrix proposal had to be amended by the addition of the crossing property i.e. a prescription of how to analytically continue par-

ticle momenta on the complex mass shell in order to relate matrix elements of local operators between incoming ket and outgoing bra states with a fixed total sum of incoming and outgoing particles as different boundary values of one analytic "masterfunction". In physical terms crossing allows to relate matrix elements describing real particle creation with particles in both the incoming ket- and outgoing bra-states to the vacuum polarization matrix elements where the ket-state (or the bra state) is the vacuum vector. 
Whereas Heisenberg's requirements of Poincaré invariance, unitarity and cluster factorization on a relativistic S-matrix can also be implemented in a "direct particle interaction" scheme [26] 27], the implementation of crossing is conceptually related to the presence of vacuum polarization for which QFT with its micro-causality is the natural arena.

The LSZ time-dependent scattering theory and the associated reduction formalism relates such a matrix element (referred to as a generalized formfactor) in a natural way to one in which an incoming particle becomes "crossed" into an outgoing anti-particle on the backward real mass shell; it is at this point where analytic continuation from a positive energy physical process enters. In this setting the S-matrix is the formfactor of the identity operator.

The important remark here is that the use of particle states requires the restriction of the analytic continuation to the complex mass shell ("on-shell"). It was $\operatorname{Bros}^{6}$ in collaboration with Epstein and Glaser 28] who gave the first rigorous proofs of crossing in special configurations. In the special case of the elastic scattering amplitude, the crossing of only one particle from the incoming state has to be accompanied by a reverse crossing of one of the outgoing particles in order to arrive at a physical process allowed by energy-momentum conservation ${ }^{7}$.

A derivation of crossing in the setting of QFT for general multi-particle scattering configurations and for formfactors (as one needs it for the derivation of a bootstrap-formfactor program, see later) from the general principles of local quantum physics does not yet exist. It is not clear to me whether the present state of art in algebraic QFT would permit to go significantly beyond the old but still impressive results quoted before.

The crossing property became the cornerstone of the so-called bootstrap S-matrix program and several ad hoc representations of analytic scattering amplitudes were proposed (Mandelstam representation, Regge poles...) in order to incorporate crossing in a more manageable form.

The algebraic basis of the bootstrap-formfactor program for the special family of $\mathrm{d}=1+1$ factorizable theories is the validity of a momentum space Zamolodchikov-Faddeev algebra [29]. The operators of this algebra are close to free fields in the sense that their Fourier transform is on-shell (see (8) in next section), but unlike the latter they are not local in the pointlike sense. A closer look reveals that they are localizable in the weaker sense ${ }^{8}$ of spacetime wedge regions 21 30. In fact the existence of such Fourier transformable ("tempered") wedge-localized PFGs, which implies the absence of real parti-

\footnotetext{
${ }^{6}$ Since the issue of crossing constitutes an important property of the present paper, it is particularly appropriate to dedicate this work to Jacques Bros on the occasion of his $70^{\text {th }}$ birthday.

${ }^{7}$ This crossing of a pair of particles from the in/out elastic configuration is actually the origin of the terminology "crossing" and was the main object of rigorous analytic investigations.

${ }^{8} \mathrm{An}$ operator which is localizable in a certain causally closed spacetime region is automatically localized in any larger region but not necessarily in a smaller region. The unspecific terminology "non-local" in the literature is used for any non pointlike localized field.
} 
cle creation through scattering processes [33, turns out to be the prerequisite for the success of the bootstrap-formfactor program for factorizable models in which one uses only formfactors and avoids (short-distance singular) correlation functions.

According to an old structural theorem which is based on certain analytic properties of a field theoretic S-matrix [34] 33], interaction-induces vacuum polarization without real particle creation is only possible in $\mathrm{d}=1+1$ theories. This in principle leaves the possibility of direct 3- or higher- particle elastic processes beyond two particle scattering. But an argument by Karowski based on formfactor crossing ${ }^{9}$ shows that the nonvanishing of higher connected elastic contributions would be inconsistent with the absence of real particle creation. In this sense the Z-F algebra structure, which is at the heart of factorizing models, turns out to be a consequence of special properties of modular wedge-localized PFGs, a fact which places the position of the factorizing models within general QFT into sharper focus. The crossing property of the two-particle scattering amplitude is a consistency prerequisite for the formfactor crossing. Providing a special illustration of the previous general unicity argument of inverse scattering based on crossing, the bootstrap formfactor approach associates precisely one QFT in the sense of one local equivalence class of fields (or one net of localized operator algebras) to a prescribed factorizing S-matrix.

In agreement with the philosophy underlying AQFT, which views pointlike fields as coordinatizations for generators of localized algebras, the bootstrapformfactor construction for $\mathrm{d}=1+1$ factorizing models primarily aims to determine coordinatization-independent double-cone algebras by computing intersections of wedge algebras. The nontriviality of a theory is then tantamount to the nontriviality $(\neq C 1)$ of such intersections. The computation of a basis of pointlike field generators of these algebras is analogous to (but more involved than) the construction of a basis of composites of free fields in the form of Wick polynomials. As we saw before for noninteracting theories the functorial description of the algebras (6) based on modular localization is conceptually simpler than the use of free fields and their local equivalence class of Wick-ordered composites e.g. one is not obliged to introduce a non-intrinsic Wick basis.

The crossing property is crucial for linking scattering data with off-shell operators spaces. As explained in the previous section, it relates the multiparticle component of vectors obtained by one-time application of a localized (at least wedge-localized) operator to the vacuum with the connected formfactors of that operator. It is important to note that in factorizing models crossing is not an assumption but rather follows from the properties of tempered PFGs for wedge algebras similar to crossing of formfactors for composite operators of free fields [36.

In the following some of the details of wedge-localized PFGs and their connections with the Zamolodchikov-Faddeev algebra structure are presented. In the simplest case of a scalar chargeless particle without bound states ${ }^{10}$ the wedge

\footnotetext{
${ }^{9} \mathrm{I}$ am indebted to M. Karowski for this argument.

${ }^{10} \mathrm{~A}$ situation which in case of factorizing models with variable coupling (e.g. the
} 
generators are of the form 21

$$
\begin{aligned}
\phi(x) & =\frac{1}{\sqrt{2 \pi}} \int\left(e^{i p(\theta) x(\chi)} Z(\theta)+\text { h.c. }\right) d \theta \\
Z(\theta) Z^{*}\left(\theta^{\prime}\right) & =S^{(2)}\left(\theta-\theta^{\prime}\right) Z^{*}\left(\theta^{\prime}\right) Z(\theta)+\delta\left(\theta-\theta^{\prime}\right) \\
Z(\theta) Z\left(\theta^{\prime}\right) & =S^{(2)}\left(\theta^{\prime}-\theta\right) Z\left(\theta^{\prime}\right) Z(\theta)
\end{aligned}
$$

Here $p(\theta)=m(\operatorname{ch} \theta, \operatorname{sh} \theta)$ is the rapidity parametrization of the $\mathrm{d}=1+1$ massshell and $x=r(\operatorname{sh} \chi, \operatorname{ch} \chi)$ parametrizes the right hand wedge in Minkowski spacetime. $S^{(2)}(\theta)$ is a structure function of the Z-F algebra which is a nonlocal *-algebra generalization of canonical creation/annihilation operators. The notation preempts the fact that $S^{(2)}(\theta)$ is the analytic continuation of the physical two-particle S-matrix $S^{(2)}(|\theta|)$ which via the factorization formula determines the general scattering operator $S_{\text {scat }}$ (11). The unitarity and crossing of $S_{\text {scat }}$ follows from the corresponding two-particle properties which in terms of the analytic continuation are $S^{2}(z)^{*}=S^{(2)}(-z)$ (unitarity) and $S^{(2)}(z)=S^{(2)}(i \pi-z)$ (crossing) 35. The $Z^{*}(\theta)$ operators applied to the vacuum in the natural order $\theta_{1}>\theta_{2}>\ldots>\theta_{n}$ are by definition equal to the outgoing canonical Fock space creation operators whereas the re-ordering from any other ordering has to be calculated according to the Z-F commutation relations e.g.

$$
Z^{*}(\theta) a^{*}\left(\theta_{1}\right) \ldots a^{*}\left(\theta_{n}\right) \Omega=\prod_{i=1}^{k} S^{(2)}\left(\theta-\theta_{i}\right) a^{*}\left(\theta_{1}\right) . . a^{*}\left(\theta_{k}\right) a^{*}(\theta) a^{*}\left(\theta_{k+1}\right) . . a^{*}\left(\theta_{n}\right) \Omega
$$

where $\theta<\theta_{i} i=1 . . k, \theta>\theta_{i} i=k+1, . . n$. The general Zamolodchikov-Faddeev algebra is a matrix generalization of this structure.

It is important not to identify the Fourier transform in (8) of the momentum with a localization variable. Although the $x$ in $\phi(x)$ behaves covariantly under Poincaré transformations, it is not marking a causal localization point; in fact it is a non-local variable in the sense of the standard use of this terminology ${ }^{11}$. It is however wedge-localized in the sense that the generating family of operator for the right-hand wedge $W$ Wightman-like (polynomial) algebra alg $\{\phi(f)$, supp $f \subset W\}$ commutes with the TCP transformed algebra $\operatorname{alg}\{J \phi(g) J$, suppg $\subset W\}$ which is the left wedge algebra 30]

$$
\begin{aligned}
& {[\phi(f), J \phi(g) J]=0} \\
& J=J_{0} S_{\text {scat }}
\end{aligned}
$$

Here $J_{0}$ is the TCP symmetry of the free field theory associated with $a^{\#}(\theta)$ and $S_{\text {scat }}$ is the factorizing S-matrix which on (outgoing) n-particle states has the

massive Thirring model) can always be obtained by choosing a sufficiently small coupling. Bound state poles in the physical $\theta$-strip require nontrivial changes of the algebraic formalism.

${ }^{11}$ The world local is reserved for "commuting for spacelike distances". In this work we are dealing with non-local fields which are nevertheless localized in causally complete subregions (wedges, double cones) of Minkowski spacetime. 
form

$$
S_{\text {scat }} a^{*}\left(\theta_{1}\right) a^{*}\left(\theta_{2}\right) \ldots a^{*}\left(\theta_{n}\right) \Omega=\prod_{i<j} S^{(2)}\left(\theta_{i}-\theta_{j}\right) a^{*}\left(\theta_{2}\right) \ldots a^{*}\left(\theta_{n}\right) \Omega
$$

if we identify the $a^{\#}(\theta)$ with the incoming creation/annihilation operators. It is then possible to give a rigorous proof 30 that the Weyl-like algebra generated by exponential unitaries is really wedge-localized and fulfills the BisognanoWichmann property

$$
\begin{aligned}
& \mathcal{A}(W)=\operatorname{alg}\left\{e^{i \phi(f)} \mid \operatorname{supp} f \subset W\right\} \\
& \mathcal{A}(W)^{\prime}=J \mathcal{A}(W) J=\mathcal{A}\left(W^{\prime}\right)
\end{aligned}
$$

where the dash on operator algebras is the standard notation for their von Neumann commutant and the dash on spacetime regions stands for the causal complement. Within the modular setting the relative position of the causally disjoint $A\left(W^{\prime}\right)$ depends via $S_{\text {scat }}$ on the dynamics. The operator TCP operator $J$ is the (antiunitary) "angular" part of the polar decomposition of Tomita's algebraically defined unbounded antilinear S-operator with the following characterization

$$
\begin{aligned}
S A \Omega & =A \Omega, A \in \mathcal{A}(W) \\
S & =J \Delta^{\frac{1}{2}}, \Delta^{i t}=U(\Lambda(-2 \pi t))
\end{aligned}
$$

with $\Lambda(\chi)$ being the Lorentz boost at the rapidity $\chi$.

At this point the setup looks like a relativistic quantum mechanics since the $\phi(f)$ (similar to genuine free fields if applied to the vacuum) do not generate vacuum polarization clouds. The advantage of the algebraic modular localization setting is that interaction-caused vacuum polarization is generated by algebraic intersections which is in agreement with the intrinsic definition of the notion of interaction presented in terms of PFGs in the previous section

$$
\begin{aligned}
\mathcal{A}(D) & \equiv \mathcal{A}(W) \cap \mathcal{A}\left(W_{a}^{\prime}\right)=\mathcal{A}(W) \cap \mathcal{A}\left(W_{a}\right)^{\prime} \\
D & =W \cap W_{a}^{\prime}
\end{aligned}
$$

This is the operator algebra associated with a double cone $D$ (which is chosen symmetric around the origin by intersecting suitably translated wedges and their causal complements). Note the difference from the quantization approach, where pointlike localized fields are used from the outset and the sharpening of localization of smeared products of fields is simply achieved by the classical step of restricting the spacetime support of the test functions. The problem of computing intersected von Neumann algebras is in general not only difficult (since there are no known general computational techniques) but also very unusual as compared to methods of standard quantization. There is a well-founded hope that one can solve the existence problem of factorizing models by showing the nontriviality of the intersections $A(D)$ 31. 
The problem becomes more amenable if one considers instead of operators their formfactors i.e. their matrix elements between incoming ket and outgoing bra state vectors. In the spirit of the LSZ formalism one can then make an Ansatz in form of a power series in $Z(\theta)$ and $Z^{*}(\theta) \equiv Z(\theta-i \pi)$ (corresponding to the power series in the incoming free field in LSZ theory). In a shorthand notation which combines both frequency parts we may write

$$
A=\sum \frac{1}{n !} \int_{C} \ldots \int_{C} a_{n}\left(\theta_{1}, \ldots \theta_{n}\right): Z\left(\theta_{1}\right) \ldots Z\left(\theta_{n}\right): d \theta_{1} \ldots d \theta_{n}
$$

where each integration path $C$ extends over the upper and lower part of the rim of the $(0,-i \pi)$ strip in the complex $\theta$-plane. The strip-analyticity of the coefficient functions $a_{n}$ expresses the wedge-localization of $A^{12}$. It is easy to see that these coefficients on the upper part of $C$ (the annihilation part) are identical to the vacuum polarization form factors of $A$

$$
\left\langle\Omega|A| p_{n}, . . p_{1}\right\rangle^{i n}=a_{n}\left(\theta_{1}, \ldots \theta_{n}\right), \quad \theta_{n}>\theta_{n-1}>\ldots>\theta_{1}
$$

whereas the crossing of some of the particles into the left hand bra state (see the previous section) leads to the connected part of the formfactors

$$
{ }^{\text {out }}\left\langle p_{1}, . . p_{k}|A| p_{n}, . . p_{k+1}\right\rangle_{\text {conn }}^{\text {in }}=a_{n}\left(\theta_{1}+i \pi, \ldots \theta_{k}+i \pi, \theta_{k+1}, . . \theta_{n}\right)
$$

Hence the crossing property of formfactors is encoded into the notation of the operator formalism (15) in that there is only one analytic function $a_{n}$ which describes the different possibilities of placing $\theta$ on the upper or lower rim of $C$.

The presence of bound states (poles in the physical $\theta$-strip) leads to a weakening of the wedge localization in the sense that the wedge commutativity (10) only holds between states from the subspace generated from the "elementary" states linearly related to (8). This requires considerable modifications of the algebraic formalism which goes beyond the modest aims of this paper.

The essential advantage of this algebraic formalism over the calculation of formfactors of individual fields is expected to appear if one tries to secure the existence of the theory. Whereas the conventional way via controlling Wightman functions and checking their properties appears hopelessly complicated (the mathematical control of the convergence of the formfactor series (15) has not even been achieved in simple models), the "modular nuclearity property" of wedge algebras in $\mathrm{d}=1+1$ which secures the nontriviality of the intersected algebras $A(D)$ 31] seems to be well in reach [37.

\section{Constructive aspects of lightfront holography}

In the previous sections it was shown how modular theory together with onshell concepts can be used to analyze special wedge algebras in the presence

\footnotetext{
${ }^{12}$ Compact localization leads to coefficient functions which are meromorphic outside the open strip 32 .
} 
of interactions. The constructive use was limited to the presence of so-called tempered PFGs which in turn restricted computable models to $d=1+1$ factorizing theories. In this section I will present a recent proposal which also uses modular localization ideas but tries to analyze wedge algebras in terms of (extended) chiral theories by means of "algebraic lightfront holography" (ALH). Again we limit ourselves to some intuitively accessible remarks mainly emphasizing analogies as well as differences with the standard formalism of QFT; for a more detailed mathematical description we again refer to the literature 36 .

The following comparison with the canonical formalism turns out to be helpful. The ETCR formalism tries to classify and construct QFTs by assuming the validity of canonical equal time commutation relations (ETCR). The shortcomings of that approach are well-known. Even if one ignores the fact that the ETC structure is inconsistent with the presence of strictly renormalizable interactions $^{13}$, the usefulness of the ETCR is still limited by its insensitivity with respect to interactions. One would prefer to start with a structure which is senses the presence of interactions and is capable to utilize the enormous amount of knowledge and structural richness which has been obtained in studying chiral theories by providing a concept of rich universality classes for higher dimensional QFT (instead of just one ETCR class).

Lightfront holography tries to address this imbalance by replacing the ETCR by the richer structure of (extended) chiral theories on the lightfront. Its main aim is to shift the cut between kinematics and dynamics in such a way that what has been learned by studying low dimensional theories can be used as a kinematical input for higher dimensional models.

The holographic projection turns out to map many different interacting ambient theories to the same holographic image; in this respect there is a certain similarity to the better known scale invariant short distance universality classes which are the key to the understanding of critical phenomena. But in contradistinction to scaling universality classes which change the theory to an associated massless theory, holographic projections live in the same Hilbert space as the ambient theory; in fact they just organize the spacetime aspects of a shared algebraic structure in a radically different way.

Let us briefly recall some salient points of $\mathrm{ALH}^{14}$.

ALH may be viewed as a kind of conceptually and mathematically refined "lightcone quantization" (or " $p \rightarrow \infty$ frame" description). Whereas the latter never faced up to the question of how the lightfront quantized fields are related to the original local fields i.e. in which sense the new description addresses the original problems posed by the ambient theory, the ALH is conceptually precise and mathematically rigorous on this points.

It turns out that the idea of restricting fields to the lightfront is limited to free fields and certain superrenormalizable interacting models with finite wave

\footnotetext{
${ }^{13}$ Only superrenormalizable interactions (finite wave function renormalization) as the polynomial scalar models in $d=1+1$ have fields which restrict to equl times

${ }^{14}$ We add this prefix "algebraic" in order to distinguish the present notion of holography from the gravitational holography of t'Hooft [38. More on similarities and differences between the two can be found in the concluding remarks,
} 
function renormalization. Theories with interaction-caused vacuum polarization which leads to Kallén-Lehmann spectral functions with diverging wave function renormalization do not permit lightfront restrictions for the same reason as they do not have equal time restrictions; e.g. for scalar fields on has ${ }^{15}$

$$
\begin{aligned}
\langle A(x) A(y)\rangle & =\int_{0}^{\infty} \rho\left(\kappa^{2}\right) i \Delta^{(+)}\left(x-y, \kappa^{2}\right) d \kappa^{2} \\
\left.\langle A(x) A(y)\rangle\right|_{L F} & \sim \int_{0}^{\infty} \rho\left(\kappa^{2}\right) d \kappa^{2} \delta\left(x_{\perp}-y_{\perp}\right) \int_{0}^{\infty} \frac{d k}{k} e^{-i k\left(x_{+}-y_{+}\right)}
\end{aligned}
$$

where in passing to the second line we used the correct rule for lightfront restriction; this is obviously not the naive one obtained by simply restricting the coordinates in the Kallén-Lehmann representation. To obtain the second line, which replaces the free field $\Delta^{+}$function by the transverse $\delta\left(x_{\perp}-y_{\perp}\right)$ delta function times the longitudinal chiral function in the $x_{+}$lightray variable, one starts from the free field representation in terms of momentum space creation/annihilation operators. In the $\mathrm{z}$-t wedge region this field may be parametrized in terms of rapidites $\chi, \theta$ as follows:

$$
\begin{aligned}
& A(x)=\frac{1}{(2 \pi)^{\frac{3}{2}}} \iint\left(e^{i m_{e f f} r c h(\chi-\theta)+\vec{p}_{\perp} \vec{x}_{\perp}} a^{*}(p)+h . c .\right) \frac{d \theta}{2} d p_{\perp} \\
& {\left[a(p), a^{*}\left(p^{\prime}\right)\right]=2 \delta\left(\theta-\theta^{\prime}\right) \delta\left(p_{\perp}-p_{\perp}^{\prime}\right), m_{e f f}=\sqrt{\vec{p}_{\perp}^{2}+m^{2}}} \\
& x=\left(r \sinh \chi, \vec{x}_{\perp}, \cosh \chi\right), p=\left(\cosh \chi, \vec{p}_{\perp}, m_{e f f} \sinh \theta\right)
\end{aligned}
$$

The limit $r \rightarrow 0$ together with a compensating limit $\chi=\hat{\chi}-\operatorname{lnm}_{e f f} r$ provides a finite lightfront limit in terms of the same creation/annihilation operators and hence takes place in the same Hilbert space (unlike the scaling limit used for critical phenomena) and leads to the desired result

$$
\begin{aligned}
\left.A(x)\right|_{L F} & =\frac{1}{(2 \pi)^{\frac{3}{2}}} \int_{0}^{\infty} \int\left(e^{i p_{-} x_{+}+i p_{\perp} x_{\perp}} a^{*}(p)+\text { h.c. }\right) \frac{d p_{-}}{2 p_{-}} d p_{\perp} \\
p_{-} & \simeq e^{-\theta}
\end{aligned}
$$

which yields the above formula for the two point function. The infrareddivergence in the longitudinal factor is spurious if one views the lightfront localization in the setting of modular wedge localization ${ }^{16}$. On the other hand the obstruction resulting from the large $\kappa$ divergence of the K-L spectral function (short distance regime of interaction-caused vacuum polarization) is shared with that which limits the range of validity of the ETCR formalism. But whereas

\footnotetext{
${ }^{15}$ It is important to realize that $\mathrm{LF}$ restriction is not a pointwise local procedure. This becomes clearer within the setting of modular localization.

${ }^{16}$ By re-expressing the rapidity testfunction space in terms of the $p_{+}$integration variable, one obtains the vanishing of the testfunctions at $p_{+}=0$. The same argument also shows that an additive modification of $\hat{\chi}$ (a multiplicative change of $p_{-}$) does not change the result in the appropriate test function setting.
} 
equal time restricted interacting fields in $d>1+2$ simply do not exist, there is no such limitation on the short distance properties of generalized chiral conformal fields which turn out to generate the ALH. What breaks down is the idea that these lightfront generating fields can be gotten simply by restricting the fields of the ambient theory, as was the case in the example of free fields.

It turns out that modular theory provides a useful tool to analyze the connection between the ambient theory and its holographic projection. Although the ambient theory may well be given in terms of pointlike fields and the ALH may also allow a pointlike description (see [25), there is no known direct relation between these fields. This was of course precisely the problem of lightcone quantization which remained unresolved. Even in the above interaction-free case when the restriction method in the sense of (21) works, the ALH net of algebras turns out to be nonlocal relative to the ambient algebra and hence the recovery of the ambient from the ALH involves nonlocal steps which the standard formalism cannot handle. Whereas lightcone quantization was not able to address those subtle problems, ALH solves them.

The intuitive physical basis of this algebraic approach is a limiting form of the causal closure property. Let $O$ be a spacetime region and $O^{\prime \prime}$ its causal closure (the causal disjoint taken subsequently taken twice) then the causal closure property is the following equality

$$
\mathcal{A}(\mathcal{O})=\mathcal{A}\left(\mathcal{O}^{\prime \prime}\right)
$$

In the case of free fields this abstract algebraic property ${ }^{17}$ is inherited via quantization from the Cauchy propagation in the classical setting of hyperbolic differential equations. The lightfront is a limiting case (characteristic surface) of a Cauchy surface. Each lightray which passes through $O^{\prime \prime}$ either must have passed or will pass through $O$. For the case of a $x^{0}-x^{3}$ wedge $W$ and its $x^{0}-x^{3}=0$ (upper) causal lightfront boundary $L F B(W)$ (which covers half of a lightfront) the relation

$$
\mathcal{A}(L F B(W))=\mathcal{A}(W) \subset B(H)
$$

is a limiting situation of the causal shadow property; a lightlike signal which goes through this boundary must have passed through the wedge (or in the terminology of causality, the wedge is the backward causal completion of its lightfront boundary). Classical data on the lightfront define a characteristic initial value problem and the smallest region which generates data localized in an open ambient region is half the lightfront as in (23); for any transversely not two-sided infinite extended subregion $O_{L F}$ on the lightfront, as well as for any region on the lightfront which is bounded in the lightray direction, the causal completion is trivial i.e. $O_{L F}=O_{L F}^{\prime \prime}$. This unusual behavior of the lightfront is related the fact that as a manifold with its metric structure inherited from the ambient Minkowski spacetime it is not even locally hyperbolic.

Several symmetries which the lightfront inherits from the ambient Poincaré group are obvious; it is clear that the lightlike translation together with the

\footnotetext{
${ }^{17}$ This equality is the local version of the "time slice property" [39].
} 
two transverse translation and the transverse rotation are leaving the lightfront invariant and that the longitudinal Lorentz boost, which leaves the wedge invariant, acts as a dilatation on the lightray in the lightfront. There are however two additional invariance transformations of the lightfront which are less obvious. Their significance in the ambient space is that of the two "translations" in the 3-parametric Wigner little group $E(2)$ of the light ray in the lightfront (a Euclidean subgroup of the 6-parametric Lorentz group). Projected into the lightfront these "translations" look like transverse Galilei transformations in the various $\left(x_{\perp}\right)_{i}-x_{+}$planes.

Modular concepts (in particular modular inclusions and intersections) provide a firm operator algebraic basis for the interplay between the ambient causality and the localization structure as well as the 7-parametric symmetry of the lightfront holography ${ }^{18}$. Among the many structural consequences we only collect those which are important for the constructive use of holography:

- The Poincaré group $P(4)$ and hence also the 7-parametric subgroup $G_{L F} \subset$ $P(4)$ which leaves the lightfront invariant are of modular origin. The full lightfront symmetry is much larger and includes the Moebius group extension of the 2-parametric longitudinal translation-dilation group which is also of modular origin.

- The lightfront algebra has no vacuum fluctuations in transverse direction i.e. the operator algebra of an longitudinal infinitely extended cylindrical region $\Xi=\left\{x_{\perp} \in Q,-\infty<x_{+}<\infty\right\}$ with finite transverse extension $Q$ is a tensor factor of the full lightfront algebra which is identical to the full ambient algebra $B(H)$

$$
\mathcal{A}(L F)=B(H)=\mathcal{A}(\Xi) \otimes \mathcal{A}(\Xi)^{\prime}, \mathcal{A}(\Xi)^{\prime}=\mathcal{A}\left(\Xi^{\prime}\right)
$$

In longitudinal direction the cyclicity of the vacuum (the Reeh-Schlieder property) prevents such a factorization i.e. the lightfront holography "squeezes" the field theoretic vacuum fluctuation into the lightray direction so that the transverse structure becomes purely quantum mechanical. As a result of the Moebius covariance along the lightray and the quantum mechanical factorization in transverse direction the lightfront holography has the structure of an (quantum mechanically) extended chiral QFT.

For the derivation of the local net structure of the lightfront theory in the longitudinal and transverse directions we refer to 36 40; this is the part which requires the use of modular localization concepts (modular inclusions and modular intersections of wedge algebras, relative commutants) which differ significantly from concepts of the standard approach to QFT. The following remarks are only intended to facilitate understanding and highlight some consequences.

\footnotetext{
${ }^{18}$ For the inverse holography the information from the fundamental lightfront inclusion $A(L F(W)) \subset_{G_{L F}} A(L F)=B(H)$ has to be complemented by the action of the $x_{-}$translation (similar to the Hamiltonian input in the ETCR approach).
} 
Although there is presently no rigorous proof, the structural analogy of the lightfront holographic projection with chiral theory leads one to expect that similar group theoretical arguments as in 41 provide the existence of covariant pointlike generators. In cases where they exist, their commutation relations are severely restricted; the transverse quantum mechanical nature only permits a delta function without derivative and the balance in the scaling dimensions restricts the longitudinal singularity structure to that of Lie fields known from chiral current or W-algebras.

$$
\begin{aligned}
& {\left[\psi_{i}\left(x_{\perp}, x_{+}\right), \psi_{j}\left(x_{\perp}^{\prime}, x_{+}^{\prime}\right)\right]=} \\
& =\delta\left(x_{\perp}-x_{\perp}^{\prime}\right)\left\{\delta^{\left(n_{i j}\right)}\left(x_{+}-x_{+}^{\prime}\right)+\sum_{k} \delta^{\left(n_{i j k}\right)}\left(x_{+}-x_{+}^{\prime}\right) \psi_{k}\left(x_{\perp}, x_{+}\right)\right\}
\end{aligned}
$$

As in the pure chiral case one may hope for rational situations in which there exists a finite set of generating fields.

The difficult part of a constructive proposal of the lightfront holography is the "inverse holography" i.e. the reconstruction of an ambient theory from its holographic projection. Apart from the interaction-free case which is characterized by a c-number commutator the kinematical holographic information is insufficient. The analogy with the canonical formalism suggests to expect that the action of the $x_{-}$lightray translation on the lightfront net or on its generating fields (25) should select a particular ambient model from the holographic equivalence class.

In the remainder of this section contains some comments on the inverse holography of factorizing models, where as a result of the two-dimensionality the transverse structure is absent and the holographic projection is a bona fide chiral theory. The on-shell aspect of covariant PFG generators for wedge algebras (8) trivializes the passing between the ambient theory and its holographic projection; within the setting of factorizing models the holographic inversion is unique and amounts to representing the action of the $x_{-}$translation (similar to the case of free fields) by the multiplication with $e^{i p_{+} x_{-}}, p_{+}=\frac{m^{2}}{p_{-}}$in the formfactor representation (15). The reason for this uniqueness is that the covariance property of the particle-like Z-F creation/annihilation operators implicitly fix the transformation properties of the full Poincaré group i.e. including the LF-changing transformations beyond $G_{L F}$.

The holographic restriction of factorizing models also highlights a new interesting aspect of chiral theories. At least those chiral models which originate in this way permit a formal representation in terms of PFGs (15) inherited from ambient theory. Although this basis of $Z^{\#}(\theta)$ operators looses its particle interpretation in the chiral holographic projection, it still continues to provide an unexpected simple "on-shell representation" simplicity for these chiral algebras. In this representation the Moebius rotations applied to states $Z^{*}(\theta) \Omega$ "dress" the latter with a vacuum polarization cloud. Again we refer for more details to [36 40]. Analogous to the free field case the inverse holography in this particu- 
lar representation just amounts to multiplication of the formfactors $a_{n}$ with the appropriate $e^{i p_{+} x_{-}}$translation factors.

This invere holography also raises the interesting question about the possible dynamical role of modular generated non-local ambient symmetries beyond the local vacuum preserving Poincaré transformations. This is part of a quest for a more profound future understanding of the relation between particle aspects of the ambient theory and chiral field aspects of its holographic projection.

We conclude with some remarks about the difference in the underlying philosophy as compared to the standard approach to QFT which is based on quantizing classical field theories i.e. on the idea that important models of particle physics can be constructed by subjecting the classical Lagrangian formalism to quantization rules. This setting leads to a finite number of possibilities of renormalizable local coupling between higher dimensional $(d \geq 1+2)$ covariant fields which contains the important standard gauge theory model of electro-weak interactions.

The modular based approach advocated here disposes of the parallelism to classical field theories; instead of quantizing concrete classical field models it aims at a classifying of models according to their intrinsic algebraic structure. The prototype situation is that of chiral models on the lightray which are classified by their Lie-type commutation structure or alternatively by analyzing the possible modular position of three MA. The underlying philosophy is that of universality classes as it is successfully used in the condensed matter physics of critical phenomena. Instead of trying to find a unique model of particle physics by quantizing a selected classical Lagrangian, one classifies holographic equivalence classes and refines the search for the best mathematical descrip-

tion of particle physics in terms of local quantum theory by adding additional dynamical informations.

\section{References}

[1] H.-J. Borchers, J. Math. Phys. 41, (2000) 3604

[2] R. Haag, N. Hugenholtz and M. Winnink, Commun. Math. Phys. 5, (1967) 215

[3] R. Haag, Local Quantum Physics, Springer 1996

[4] J. Norton, The Hole Argument, The Stanford Encyclopedia of Philosophy (spring 2004 edition), Edward N. Zalta ed., http://plato.stanford.edu/archives/spr2004/entries/spacetime-holearg/

[5] J. J. Bisognano and E. H. Wichmann, J. Math. Phys. 16, (1975) 985

[6] A. Connes, Ann. Inst. Fourier 24, (1974) 121

[7] W. Driessler, Commun. Math. Phys. 53, (1977) 295 
[8] S. J. Summers, Tomita-Takesaki Modular Theory, to appear in the Encyclopedia of Mathematical Physics, Elsevier Publ.

[9] J. Yngvason, The role of type III factors in quantum field theory, math-ph/0411058

[10] R. Brunetti, K. Fredenhagen and R. Verch, Commun. Math. Phys. 237, (2003) 31

[11] R. Kaehler and H.-W. Wiesbrock, J. Math. Phys. 42, (2001) 74

[12] R. Brunetti, D. Guido and R. Longo, Rev. Math. Phys. 14, (2002) 759

[13] J. Mund, Ann. H. Poinc. 2, (2001) 907

[14] L. Fassarella and B. Schroer, J. Phys. A: Math. Gen.35, (2002) 9123

[15] J. Mund, B. Schroer and J. Yngvason, String-localized Quantum Fields and Modular Localization, in preparation

[16] M. Rieffel and A. van Daele, Pacific J. of Math. 69, (1977) 187

[17] J. Mund, B. Schroer and J. Yngvason, Phys. Lett. B 596, (2004) 156

[18] J.Math.Phys. 44, (2003) 2037

[19] M. Takesaki, Tomita's Theory of Modular Hilbert Algebras and its Applications, Lecture Notes in Mathematics Vol. 128, Springer-Verlag Berlin, Heidelberg, New York (1970)

[20] P. Leyland, J. Roberts and D. Testard, Duality for quantum free fields, unpublished notes 1978

[21] B. Schroer, Annals of Physics 295, (1999) 190

[22] R. F. Streater and A. S. Wightman, PCT Spin\&Statistics and All That, Benjamin 1964

[23] O. Steinmann, Commun. Math. Phys. 87, (1982) 259

[24] H.-W. Wiesbrock, Comm. Math. Phys. 158, (1993) 537

[25] W. Heisenberg, Zeitschr. fuer Naturforschung 1, (1946) 608

[26] F. Coester, Helv. Physica Acta 38, (1965) 7

[27] B. Schroer, An anthology of non-local QFT and QFT on noncommutative spacetime, hep-th/040510

[28] J. Bros and H. Epstein and V. Glaser, Commun. Math. Phys. 1, (1965) 240J. Bros and H. Epstein and V. Glaser, Commun. Math. Phys. 1, (1965) 240 
[29] A. B. Zamolodchikov, Int. J. of Mod. Phys. A1, (1989) 1235

[30] G. Lechner, Lett. Math. Phys. 64, (2003) 137

[31] D. Buchholz and G. Lechner, Modular Nuclearity and Localization, math-ph/0402072

[32] H. M. Babujian, A. Fring, M. Karowski and A. Zappletal, Nucl. Phys. B538, (1999) 535

[33] H. J. Borchers, D. Buchholz and B. Schroer, Commun. Math. Phys. 219, (2001) 125, hep-th/0003243

[34] S. Åks, Journ. Math. Phys. 6, (1965) 516

[35] M. Karowski, H.J Thun, T. T. Truong and P Weisz, Phys. Lett. 67 B (1977) 321

[36] B. Schroer, Constructive proposals for QFT based on the crossing property and on lightfront holography, hep-th/0406016

[37] G. Lechner, On the Existence of Local Observables in Theories with a Factorizing S-Matrix, math-ph/0405062

[38] G. 't Hooft, in Salam-Festschrift, A. Ali et al. eds., World Scientific (1993) 284

[39] R. Haag and B. Schroer, J. Math. Phys. 5, (1962) 248

[40] J. Barata, J. Mund and B. Schroer, in preparation

[41] M. Jörss, Lett. Math.Phys. 38, (1996) 257 